\title{
Numerical Sequence of Borane Series
}

\section{ENOS MASHEIJA KIREMIRE}

Department of Chemistry and Biochemistry, University of Namibia, Private Bag 13301, Windhoek, Namibia.

http://dx.doi.org/10.13005/ojc/300317

(Received: July 01, 2014; Accepted: August 08, 2014)

\begin{abstract}
A table of hydroborane families has been created. The table links boranes of different families(homologous series) and members of the same family based on $\mathrm{k}$ number. The table is useful deducing straight away whether a borane( molecular formula) is closo, nido or arachno or something else. The table also indicates that boranes are formed according to natural periodic function (arithmetical progression). The empirical formula utilized is extremely versatile, simple and based on the principle of Nobel gas configuration. It could be used in both simple and complex boranes and carboranes. The closo members which portray characteristic shapes also have characteristic $\mathrm{k}_{1}$ numbers.
\end{abstract}

Key words: Numerical sequence, Borane series, closo members.

\section{INTRODUCTION}

As a result of the carbon (C) atom to catenate and also form strong bonds with a hydrogen atom, it is able to generate a vast range of families of hydrocarbons. Some of the common families may be given by the formulas

$\mathrm{F}_{1}=\mathrm{C}_{\mathrm{n}} \mathrm{H}_{2 n+2}$ (alkanes), $\mathrm{F}_{2}=\mathrm{C}_{\mathrm{n}} \mathrm{H}_{2 \mathrm{n}}$ (alkenes), and $\mathrm{F}_{3}=$ $\mathrm{C}_{\mathrm{n}} \mathrm{H}_{2 n-2}$ (alkynes).

For each of these families, the carbon atom obeys the octet (8) rule which has its base in a noble gas configuration of neon $(\mathrm{Ne})$. On the other hand the boron atom (B) which is next to the carbon atom one valence electron less, mimics the carbon atom to produce boron hydrides (boranes) in an attempt to obey the octet rule. For instance, diborane $\mathrm{B}_{2} \mathrm{H}_{6}=(\mathrm{BH})_{2} \mathrm{H}_{4}$ may regarded as attempting to mimic $\mathrm{C}_{2} \mathrm{H}_{4}$ since $(\mathrm{BH})$ fragment is electronically equivalent to $(C)$ atom. Indeed, their shapes could be viewed as indicated in the sketch of Fig. 1. The two hydrogen atoms indicated by dots in the two bonds, can be considered to donate two electrons into the diboranemolecule so as to enable the boron atoms to satisfy the octet rule. In reality, it should be noted that diborane has two banana bonds of bridging $\mathrm{H}$ atoms.

\section{Borane series}

Just like hydrocarbons, the known boron 
<smiles>C=C</smiles><smiles>[H][R5]=C</smiles>

Fig. 1: The relationship between $\mathrm{C}_{2} \mathrm{H}_{24}$ and $\mathrm{B}_{2} \mathrm{H}_{6}$

hydrides have been grouped into families. These families are known as CLOSO, NIDO, ARACHNO and HYPO and the rare one KLADO. These families may be represented by the formulas given below.

$\mathrm{G}_{0}=\mathrm{B}_{\mathrm{n}} \mathrm{H}_{\mathrm{n}}, \mathrm{G}_{1}=\mathrm{B}_{\mathrm{n}} \mathrm{H}_{\mathrm{n}+2}$ (CLOSO), $\mathrm{G}_{2}=$ $B_{n} H_{n+4}\left(\right.$ NIDO) , $G_{3}=B_{n} H_{n+6}$ (ARACHNO), $G_{4}=B_{n} H_{n+8}$ (HYPO), $G_{5}=B_{n} H_{n+10}(P=K L A D O), G_{6}=B_{n} H_{n+12}$ (Q), $G_{7}=B_{n} H_{n+14}(R)$. These series (families) are given in Table 1. Each member of the family has been given a number $\left(k_{1}\right.$ value). The $k_{1}$ values are calculated from the empirical formula $k_{1}=1 / 2(E-V)$, where $E$ = sum of octet electrons and $V=$ sum of valence electrons in the cluster. This empirical formula has been discussed in our previous publication $\mathbf{s}^{1-2}$. What is interesting about the empirical formula is that it predicts a quadruple bond for $\mathrm{C}_{2}$ diatomic molecule as found from high level theoretical calculation methods $^{3-4}$.

\section{Analysis of $k_{1}$ values}

The $k_{1}$ numbers do vary from family to family and also within the members of the same boron family. A movement from one family member to the next family member (horizontally, similar to a period in the periodic table), the $k_{1}$ number varies by 1. On the other hand a movement from one member to the next member of the same family ( similar to a group in the periodic table), the $k_{1}$ number varies by 2. Clearly these are simple arithmetical series. The following examples illustrate this point.

Across families: $\mathrm{B}_{6} \mathrm{H}_{6}(12), \mathrm{B}_{6} \mathrm{H}_{8}(11)$, $\mathrm{B}_{6} \mathrm{H}_{10}(10), \mathrm{B}_{6} \mathrm{H}_{12}(9), \mathrm{B}_{6} \mathrm{H}_{14}(8)$; (simply add or subtract $2 \mathrm{H}$ atoms)and across members of the same family: $\mathrm{B}_{5} \mathrm{H}_{7}(9), \mathrm{B}_{6} \mathrm{H}_{8}(11), \mathrm{B}_{7} \mathrm{H}_{9}(13), \mathrm{B}_{8} \mathrm{H}_{10}(15)$,

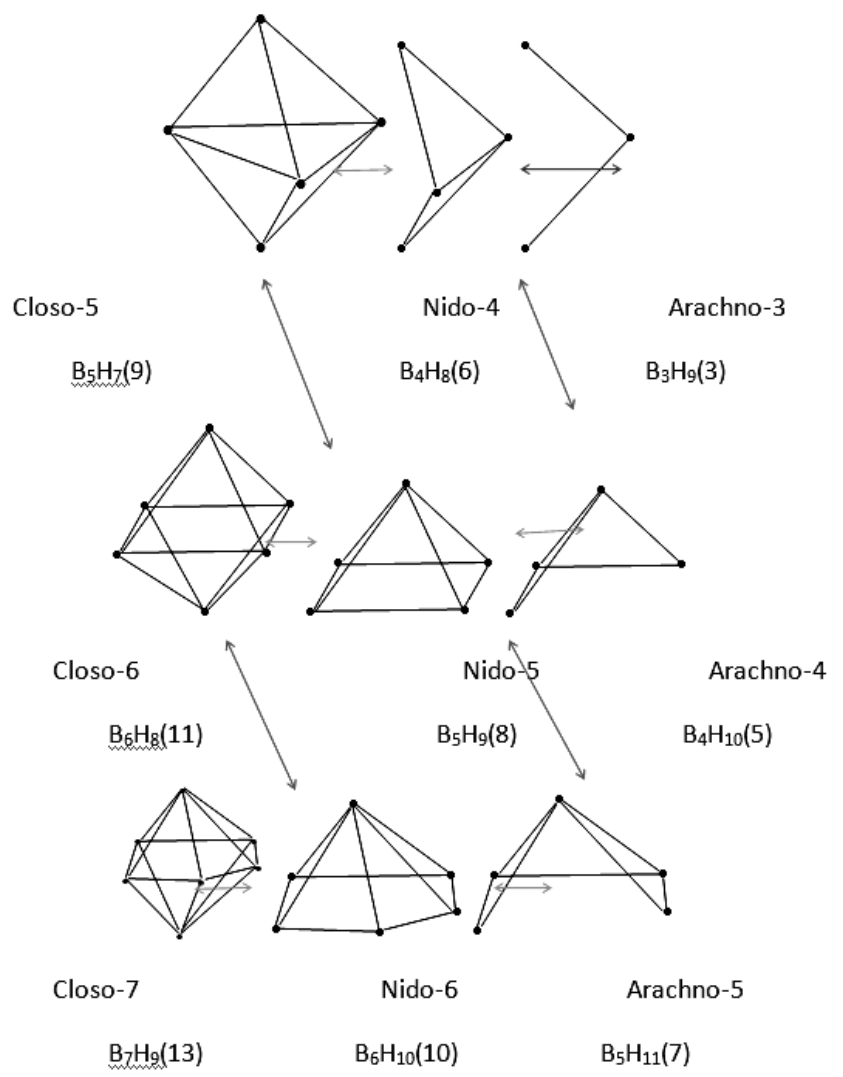

Fig. 2: Geometrical Relationship corresponding to the Diagonal Relationship of k Values of Boranes 


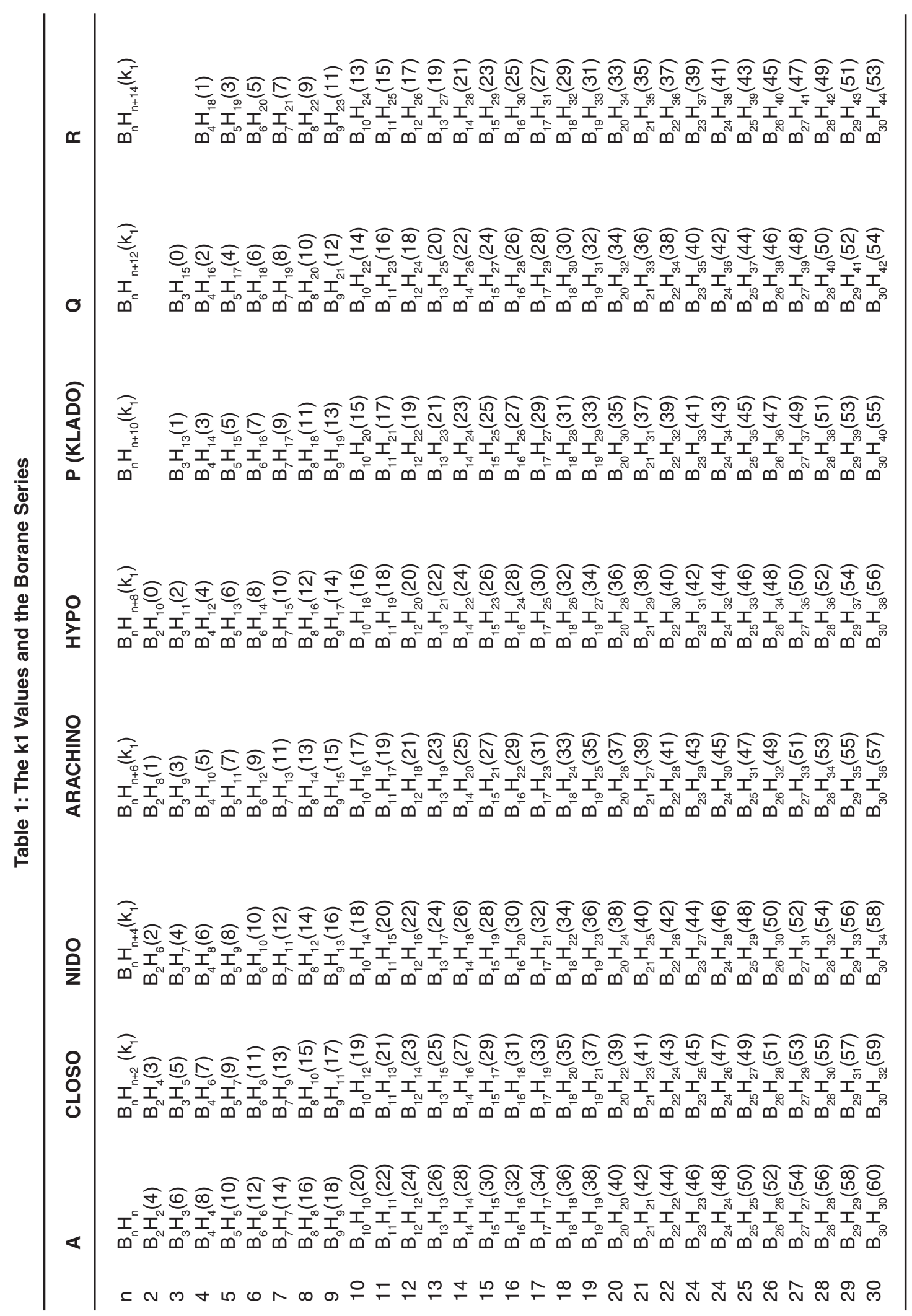


Table 2: Diagonal Relationship of $\mathbf{k}$ Values ofBoranes for $\mathbf{n}=\mathbf{2}$ to 12

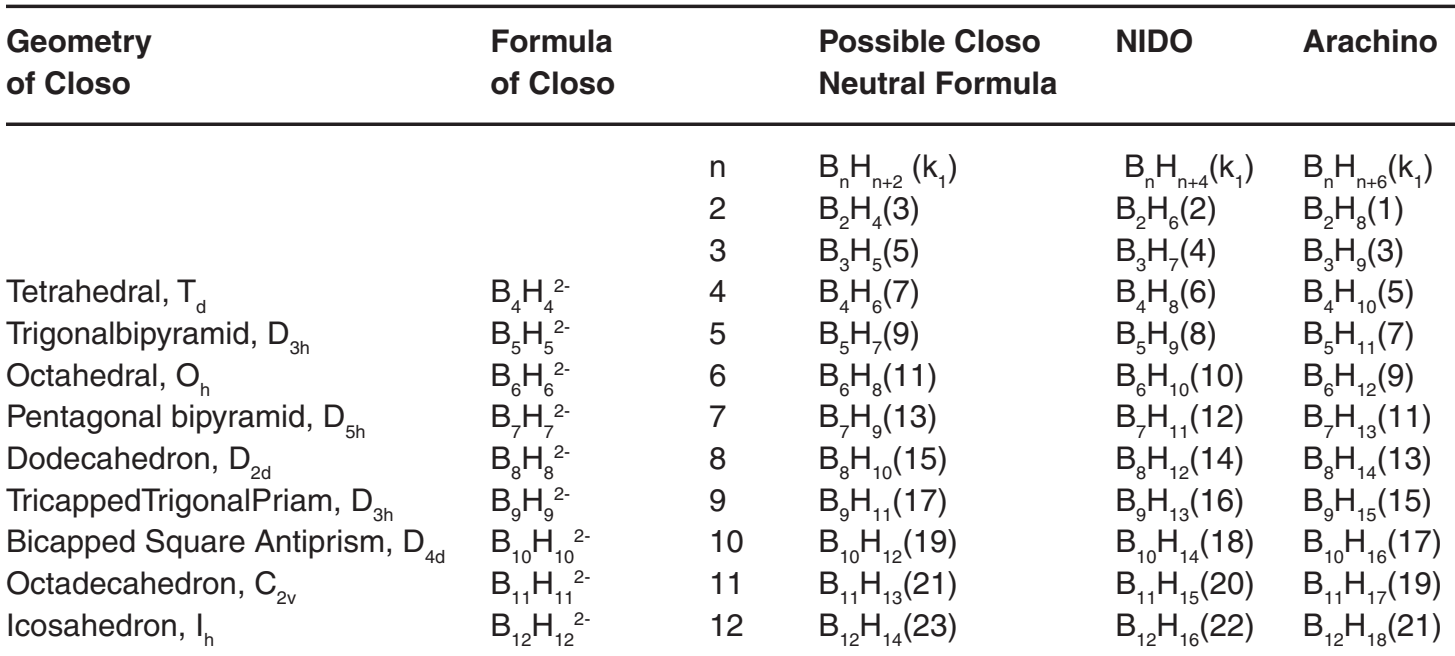

Table 3: Geometrical Relationship corresponding to the Diagonal Relationship of $\mathbf{k}$ Values of Boranes

\begin{tabular}{llll}
\hline $\mathrm{n}$ & CLOSO & $\mathrm{NIDO}$ & $\mathrm{ARACHNO}$ \\
& $\mathrm{B}_{\mathrm{n}} \mathrm{H}_{\mathrm{n}+2}\left(\mathrm{k}_{1}\right)$ & $\mathrm{B}_{\mathrm{n}+4}\left(\mathrm{k}_{1}\right)$ & $\mathrm{B}_{\mathrm{n}+6}\left(\mathrm{k}_{1}\right)$ \\
& $\mathrm{n}$ & $\mathrm{n}-1$ & $\mathrm{n}-2$ \\
3 & $\mathrm{~B}_{3} \mathrm{H}_{5}(5)$ & $\mathrm{B}_{2} \mathrm{H}_{6}(2)$ & \\
4 & $\mathrm{~B}_{4} \mathrm{H}_{6}(7)$ & $\mathrm{B}_{3} \mathrm{H}_{7}(4)$ & $\mathrm{B}_{2} \mathrm{H}_{8}(1)$ \\
5 & $\mathrm{~B}_{5} \mathrm{H}_{7}(9)$ & $\mathrm{B}_{4} \mathrm{H}_{8}(6)$ & $\mathrm{B}_{3} \mathrm{H}_{9}(3)$ \\
6 & $\mathrm{~B}_{6} \mathrm{H}_{8}(11)$ & $\mathrm{B}_{5} \mathrm{H}_{9}(8)$ & $\mathrm{B}_{4} \mathrm{H}_{10}(5)$ \\
7 & $\mathrm{~B}_{7} \mathrm{H}_{9}(13)$ & $\mathrm{B}_{6} \mathrm{H}_{10}(10)$ & $\mathrm{B}_{5} \mathrm{H}_{11}(7)$ \\
8 & $\mathrm{~B}_{8} \mathrm{H}_{10}(15)$ & $\mathrm{B}_{7} \mathrm{H}_{11}(12)$ & $\mathrm{B}_{6} \mathrm{H}_{12}(9)$ \\
9 & $\mathrm{~B}_{9} \mathrm{H}_{11}(17)$ & $\mathrm{B}_{8} \mathrm{H}_{12}(14)$ & $\mathrm{B}_{7} \mathrm{H}_{13}(11)$ \\
10 & $\mathrm{~B}_{10} \mathrm{H}_{12}(19)$ & $\mathrm{B}_{9} \mathrm{H}_{13}(16)$ & $\mathrm{B}_{8} \mathrm{H}_{14}(13)$ \\
11 & $\mathrm{~B}_{11} \mathrm{H}_{13}(21)$ & $\mathrm{B}_{10} \mathrm{H}_{14}(18)$ & $\mathrm{B}_{9} \mathrm{H}_{15}(15)$ \\
12 & $\mathrm{~B}_{12} \mathrm{H}_{14}(23)$ & $\mathrm{B}_{11} \mathrm{H}_{15}(20)$ & $\mathrm{B}_{10} \mathrm{H}_{16}(17)$ \\
\hline
\end{tabular}

$\mathrm{B}_{9} \mathrm{H}_{11}(17), \mathrm{B}_{10} \mathrm{H}_{12}(19), \mathrm{B}_{11} \mathrm{H}_{13}(21), \mathrm{B}_{12} \mathrm{H}_{14}(23)$ ( simply add or subtract $\mathrm{BH}$ fragment). A large number of hypothetical neutral boranes expected from the formulas have never been synthesized. For instance, the neutral members of the closo family, $\mathrm{B}_{5} \mathrm{H}_{7}, \mathrm{~B}_{6} \mathrm{H}_{8}$, $\mathrm{B}_{7} \mathrm{H}_{9}, \mathrm{~B}_{8} \mathrm{H}_{10}, \mathrm{~B}_{9} \mathrm{H}_{11}, \mathrm{~B}_{10} \mathrm{H}_{12}, \mathrm{~B}_{11} \mathrm{H}_{13}$, and $\mathrm{B}_{12} \mathrm{H}_{14}$ have

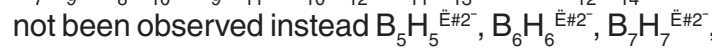

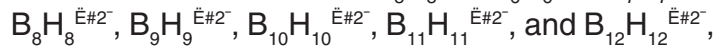
are well known. In Table 1, it hasbeen found easier to explain the series by assuming that all boranes are neutral. For instance to test to which family a hydroborane belongs, $\mathrm{B}_{2} \mathrm{H}_{7} \mathrm{E \#}^{-}$is regarded as $\mathrm{B}_{2} \mathrm{H}_{8}$ and $\mathrm{B}_{5} \mathrm{H}_{8}{ }^{\ddot{E} \#-}$ as $\mathrm{B}_{5} \mathrm{H}_{9}$. A collection sample of boranes have been classified based on Table 1 and these are presented in Table 5.

Table 4: Geometrical Relationship in Fig. 1 expressed in $\mathbf{k}_{1}$ values

\begin{tabular}{lllll}
\hline & Starting Cluster & Closo & NIDO & Arachno \\
\hline \multirow{3}{*}{$\mathrm{B} \mathrm{H}$} & $-\mathrm{B}+\mathrm{H}$ & & & \\
& $\mathrm{B}_{4} \mathrm{H}_{6} \quad\left(\mathrm{~B}_{4} \mathrm{H}_{4}{ }^{2-}\right)$ & 7 & 4 & 1 \\
& $\mathrm{~B}_{5} \mathrm{H}_{7} \quad\left(\mathrm{~B}_{5} \mathrm{H}_{5}{ }^{2-}\right)$ & 9 & 6 & 3 \\
& $\mathrm{~B}_{6} \mathrm{H}_{8}\left(\mathrm{~B}_{6} \mathrm{H}_{6}{ }^{2-}\right)$ & 11 & 8 & 5 \\
& $\mathrm{~B}_{7} \mathrm{H}_{9}\left(\mathrm{~B}_{7} \mathrm{H}_{7}{ }^{2-}\right)$ & 13 & 10 & 7 \\
$\mathrm{~B}_{8} \mathrm{H}_{10}\left(\mathrm{~B}_{8} \mathrm{H}_{8}{ }^{2-}\right)$ & 15 & 12 & 9 \\
$\mathrm{~B}_{9} \mathrm{H}_{11}\left(\mathrm{~B}_{9} \mathrm{H}_{9}{ }^{2-}\right)$ & 17 & 14 & 11 \\
$\mathrm{~B}_{10} \mathrm{H}_{12}\left(\mathrm{~B}_{10} \mathrm{H}_{10}{ }^{2-}\right)$ & 19 & 16 & 13 \\
$\mathrm{~B}_{11} \mathrm{H}_{13}\left(\mathrm{~B}_{11} \mathrm{H}_{11}{ }^{2-}\right)$ & 21 & 18 & 15 \\
$\mathrm{~B}_{12} \mathrm{H}_{14}\left(\mathrm{~B}_{12} \mathrm{H}_{12}{ }^{2-}\right)$ & 23 & 20 & 17 \\
& & &
\end{tabular}


Table 5: Selected Examples of Boranes for Classification Using Table 1

\begin{tabular}{ccc}
\hline$B_{n}$ & Examples & Proposed Classification \\
\hline 1 & & \\
2 & $\mathrm{~B}_{2} \mathrm{H}_{6}, \mathrm{~B}_{2} \mathrm{H}_{8}$ & $\mathrm{NAR}$ \\
3 & $\mathrm{~B}_{3} \mathrm{H}_{9}$ & $\mathrm{AR}$ \\
4 & $\mathrm{~B}_{4} \mathrm{H}_{8}$, & $\mathrm{N}$ \\
& $\mathrm{B}_{4} \mathrm{H}_{10}$ & $\mathrm{AR}$ \\
5 & $\mathrm{~B}_{5} \mathrm{H}_{7}, \mathrm{~B}_{5} \mathrm{H}_{9}$, & $\mathrm{CN}$ \\
& $\mathrm{B}_{5} \mathrm{H}_{11}$ & $\mathrm{AR}$ \\
& $\mathrm{B}_{5} \mathrm{H}_{13}$ & $\mathrm{H}$ \\
6 & $\mathrm{~B}_{6} \mathrm{H}_{8}$, & $\mathrm{C}$ \\
& $\mathrm{B}_{6} \mathrm{H}_{10} \mathrm{~B}_{6} \mathrm{H}_{12}$ & $\mathrm{NAR}$ \\
7 & $\mathrm{~B}_{7} \mathrm{H}_{9} \mathrm{~B}_{7} \mathrm{H}_{13}$ & $\mathrm{C}$ \\
8 & $\mathrm{~B}_{8} \mathrm{H}_{10}$, & $\mathrm{C}$ \\
& $\mathrm{B}_{8} \mathrm{H}_{12}$, & $\mathrm{N}$ \\
& $\mathrm{B}_{8} \mathrm{H}_{14}$, & $\mathrm{AR}$ \\
& $\mathrm{B}_{8} \mathrm{H}_{16}$ & $\mathrm{H}$ \\
& $\mathrm{B}_{8} \mathrm{H}_{18}$ & $\mathrm{P}$ \\
9 & $\mathrm{~B}_{9} \mathrm{H}_{11}$ & $\mathrm{C}$ \\
& $\mathrm{B}_{9} \mathrm{H}_{13}$, & $\mathrm{N}$ \\
& $\mathrm{B}_{9} \mathrm{H}_{15}$ & $\mathrm{AR}$ \\
10 & $\mathrm{~B}_{10} \mathrm{H}_{12}$, & $\mathrm{C}$ \\
& $\mathrm{B}_{10} \mathrm{H}_{14}$, & $\mathrm{N}$ \\
& $\mathrm{B}_{10} \mathrm{H}_{16}$, & $\mathrm{H}$ \\
& $\mathrm{B}_{10} \mathrm{H}_{18}$ & \\
\hline
\end{tabular}

\section{Diagonal relationship within the families}

The diagonal relationship within boranec families can readily be discerned from Table 1. The examples include $\mathrm{B}_{5} \mathrm{H}_{7}(9)^{\prime} ! \mathrm{B}_{4} \mathrm{H}_{8}(6)^{\prime} ! \mathrm{B}_{3} \mathrm{H}_{9}(3)$; and $\mathrm{B}_{6} \mathrm{H}_{8}(11)^{\prime} ! \mathrm{B}_{5} \mathrm{H}_{9}(8)$ '! $\mathrm{B}_{4} \mathrm{H}_{10}(5)$. This diagonal relationship also presented horizontally in Table 3 , is cited widely in form of shapes ${ }^{5}$ and is partially presented in Fig. 1. The $k_{1}$ numbers vary by 3 units. The diagonal relationship within boron families when set up horizontally, another diagonal relationship is detected. The variations are given in Table 4 . This relationship links up boranes with the same number of $B$ atoms but different number of hydrogen atoms from different families. For instance, $\mathrm{B}_{5} \mathrm{H}_{7}(9)$ (CLOSO)'! $\mathrm{B}_{5} \mathrm{H}_{9}(8)$ (NIDO)'! $\mathrm{B}_{5} \mathrm{H}_{11}$ (7)(ARACHNO). The $k_{1}$ values vary by 1 and therefore the shapes differ.It has also been found from theoretical studies by Yao and Hoffman ${ }^{6}$ that the boranes $\mathrm{B}_{3} \mathrm{H}_{9}, \mathrm{~B}_{4} \mathrm{H}_{12}$ and $\mathrm{B}_{6} \mathrm{H}_{18}$ have some stability with $\mathrm{B}_{6} \mathrm{H}_{18}$ being the most stable. These boranes belong to the families, ARACHNO, HYPO, and P (see Table 1) respectively. Furthermore, theoretical calculations ${ }^{7}$ indicate that the boranes $\mathrm{B}_{4} \mathrm{H}_{2}, \mathrm{~B}_{4} \mathrm{H}_{24}, \mathrm{~B}_{4} \mathrm{H}_{6}, \mathrm{~B}_{4} \mathrm{H}_{8}$ and $\mathrm{B}_{4} \mathrm{H}_{10}($ belong to the families $B, C, N, A R$ in the Table 1) are more stable than $\mathrm{B}_{4} \mathrm{H}, \mathrm{B}_{4} \mathrm{H}_{3}, \mathrm{~B}_{4} \mathrm{H}_{5}, \mathrm{~B}_{4} \mathrm{H}_{7}$, and $\mathrm{B}_{4} \mathrm{H}_{9}$ which do not belong to any of the families.

Table 1: Extended left wise

\begin{tabular}{|c|c|c|c|c|c|}
\hline & G & $F$ & $E$ & D & $\mathrm{C}_{0}$ \\
\hline $\mathrm{n}$ & $\mathrm{B}_{n} \mathrm{H}_{n-1}$ & $\mathrm{~B}_{n} \mathrm{H}_{\mathrm{n}}$ & $\mathrm{B}_{n} \mathrm{H}_{\mathrm{n}}$ & $\mathrm{BH}_{\mathrm{n}-\mathrm{H}}$ & $\mathrm{Br}_{\mathrm{n}}$ \\
\hline 1 & & & & & \\
\hline 2 & 0 & 0 & 0 & 0 & $\mathrm{~B}_{2}$ \\
\hline 3 & 0 & 0 & 0 & 0 & $\mathrm{~B}_{3}^{2} \mathrm{H}$ \\
\hline 4 & 0 & 0 & 0 & $\mathrm{~B}_{4}$ & $\mathrm{~B}_{4}^{3} \mathrm{H}_{2}$ \\
\hline 5 & 0 & 0 & 0 & $\mathrm{~B}_{5}^{4} \mathrm{H}$ & $\mathrm{B}_{5}^{4} \mathrm{H}_{3}^{2}$ \\
\hline 6 & 0 & 0 & $\mathrm{~B}_{6}$ & $\mathrm{~B}_{6}^{5} \mathrm{H}_{2}$ & $\mathrm{~B}_{6}^{5} \mathrm{H}_{4}^{3}$ \\
\hline 7 & 0 & 0 & $\mathrm{~B}_{7}^{6} \mathrm{H}$ & $\mathrm{B}_{7}^{6} \mathrm{H}_{3}^{2}$ & $\mathrm{~B}_{7}^{6} \mathrm{H}_{5}^{4}$ \\
\hline 8 & 0 & $\mathrm{~B}_{8}$ & $\mathrm{~B}_{8}^{7} \mathrm{H}_{2}$ & $\mathrm{~B}_{8} \mathrm{H}_{4}^{3}$ & $\mathrm{~B}_{8}^{7} \mathrm{H}_{6}^{5}$ \\
\hline 9 & 0 & $\mathrm{~B}_{0}^{8} \mathrm{H}$ & $\mathrm{B}_{9}^{8} \mathrm{H}_{3}^{2}$ & $\mathrm{~B}_{0}^{8} \mathrm{H}_{5}^{4}$ & $\mathrm{~B}_{9}^{8} \mathrm{H}_{7}^{6}$ \\
\hline 10 & $\mathrm{~B}_{1}$ & $\mathrm{~B}_{10} \mathrm{H}_{2}$ & $\mathrm{~B}_{10}^{9} \mathrm{H}_{4}$ & $\mathrm{~B}_{10} \mathrm{H}_{6}$ & $\mathrm{~B}_{10} \mathrm{H}_{8}$ \\
\hline 11 & $\mathrm{~B}_{11}^{10} \mathrm{H}$ & $\mathrm{B}_{11}^{10} \mathrm{H}_{3}^{2}$ & $\mathrm{~B}_{11}^{10} \mathrm{H}_{5}^{4}$ & $\mathrm{~B}_{11}^{10} \mathrm{H}_{7}^{6}$ & $\mathrm{~B}_{11}^{10} \mathrm{H}_{9}^{8}$ \\
\hline 12 & $\mathrm{~B}_{12}^{11} \mathrm{H}_{2}$ & $\mathrm{~B}_{12}^{11} \mathrm{H}_{4}^{3}$ & $\mathrm{~B}_{12}^{11} \mathrm{H}_{6}^{5}$ & $\mathrm{~B}_{12}^{11} \mathrm{H}_{8}^{\prime}$ & $\mathrm{B}_{12}^{11} \mathrm{H}_{10}^{9}$ \\
\hline 13 & $\mathrm{~B}_{13}^{12} \mathrm{H}_{3}^{2}$ & $\mathrm{~B}_{13}^{12} \mathrm{H}_{5}^{4}$ & $\mathrm{~B}_{13}^{12} \mathrm{H}_{7}^{6}$ & $\mathrm{~B}_{13}^{12} \mathrm{H}_{9}^{8}$ & $\mathrm{~B}_{13}^{12} \mathrm{H}_{11}^{10}$ \\
\hline 14 & $\mathrm{~B}_{14}^{13} \mathrm{H}_{4}^{3}$ & $\mathrm{~B}_{14}^{13} \mathrm{H}_{6}^{5}$ & $\mathrm{~B}_{14}^{13} \mathrm{H}_{8}^{\prime}$ & $\mathrm{B}_{14}^{13} \mathrm{H}_{10}^{9}$ & $\mathrm{~B}_{14}^{13} \mathrm{H}_{12}$ \\
\hline 15 & $\mathrm{~B}_{15}^{14} \mathrm{H}_{5}^{4}$ & $\mathrm{~B}_{15}^{14} \mathrm{H}_{7}^{6}$ & $\mathrm{~B}_{15}^{14} \mathrm{H}_{9}^{8}$ & $\mathrm{~B}_{15}^{14} \mathrm{H}_{11}^{10}$ & $\mathrm{~B}_{15}^{14} \mathrm{H}_{13}$ \\
\hline 16 & $\mathrm{~B}_{16}^{10} \mathrm{H}_{6}^{5}$ & $\mathrm{~B}_{16}^{10} \mathrm{H}_{8}^{\prime}$ & $\mathrm{B}_{16}^{15} \mathrm{H}_{10}^{9}$ & $\mathrm{~B}_{16}^{15} \mathrm{H}_{12}^{11}$ & $\mathrm{~B}_{16}^{15} \mathrm{H}_{14}^{13}$ \\
\hline 17 & $\mathrm{~B}_{17}^{10} \mathrm{H}_{7}^{\circ}$ & $\mathrm{B}_{17}^{10} \mathrm{H}_{9}^{\circ}$ & $\mathrm{B}_{17} \mathrm{H}_{11}^{10}$ & $\mathrm{~B}_{17}^{10} \mathrm{H}_{13}^{12}$ & $\mathrm{~B}_{17} \mathrm{H}_{15}$ \\
\hline 18 & $\mathrm{~B}_{18}^{\prime \prime} \mathrm{H}_{8}^{\prime}$ & $\mathrm{B}_{18} \mathrm{H}_{10}^{\circ}$ & $\mathrm{B}_{18} \mathrm{H}_{12}$ & $\mathrm{~B}_{18} \mathrm{H}_{14}^{13}$ & $\mathrm{~B}_{18} \mathrm{H}_{16}$ \\
\hline 19 & $\mathrm{~B}_{19}^{18} \mathrm{H}_{9}^{8}$ & $\mathrm{~B}_{19}^{18} \mathrm{H}_{11}^{10}$ & $\mathrm{~B}_{19}^{\mathrm{B}} \mathrm{H}_{13}^{\mathrm{IL}}$ & $\mathrm{B}_{19}^{18} \mathrm{H}_{15}^{14}$ & $\mathrm{~B}_{19} \mathrm{H}_{17}$ \\
\hline 20 & $\mathrm{~B}_{20} \mathrm{H}_{10}^{9}$ & $\mathrm{~B}_{20}^{19} \mathrm{H}_{12}$ & $\mathrm{~B}_{20}^{19} \mathrm{H}_{14}^{13}$ & $\mathrm{~B}_{20}^{19} \mathrm{H}_{16}^{15}$ & $\mathrm{~B}_{20} \mathrm{H}_{18}$ \\
\hline
\end{tabular}


Table 1 has been extended on the left to cover the boron families headed by symbols $\mathrm{C}_{0}$, $\mathrm{D}$, $E$, and $G$. The whole Table 1 covers the series $B_{n} H_{n-10}$ to $\mathrm{B}_{\mathrm{n}} \mathrm{H}_{\mathrm{n}+14}$

\section{CONCLUSION}

Borane series tend to occur in families just like hydrocarbons. The driving force for cluster formation is probably due to boranes attempting to obey the octet rule. A given borane that obeys the octet rule may be classified to belong to a particle family using the table. Both the known boranes and the theoretically established as being stable appear to fit into one of the families. Just as the elements of the periodic table follow a simple arithmetical progression, the boranes across the row horizontally (families- similar to the period in the periodic table)) and vertically ( within the family members- similar to a group within the periodic table) as well as diagonally (similar to the diagonal relationship of elements in the periodic table) follow simple arithmetical progression. The $k_{1}$ values are useful in organizing the boron families and their members.

\section{REFERENCES}

1. Kiremire, E. M. R.Oriental J. Chem. 2007, 32(2), 809-812.

2. Kiremire, E. M. R.;Kiremire, E. B. B. Materials Science Research India2007,4(1), 09-18.

3. Shaik, S.;Danovich, D.; Wu, W.; Su, P.; Rzepa, S.; Hiberty, P. C. Nature2012 Chemistry, 4, 195-200.

4. Su, P.;Wu, J.; Gu, J.;Wu, W.; Shaik, S.; Hiberty, P. C. J. Chem. Theor. Comput. 2011,7, 121-
130.

5. Fox, M. A.; Wade, K. Pure Appl. Chem.2003, 75(9), 1315-1323.

6. Yao, Y.; Hoffman, R. J. Am. Chem. Soc.,2011, 133, 21002-21009.

7. Böyükata, M.;özdoðana, C.; GÜvenç, Z. B. Romanian J. of Information Science and Technology 2008, 11(1), 59-70. 